\title{
Endoscopic ultrasound-guided rendezvous in benign biliary or pancreatic disorders with a 22-gauge needle and a 0.018-inch guidewire $\square$
}

\section{두)(요우}

\author{
Authors \\ Institutions \\ 1 Servicio Aparato Digestivo. Hospital Universitario del \\ Vinalopó, Elche, Alicante, Spain \\ 2 Unidad de Endoscopia. Servicio de Aparato Digestivo, \\ Hospital General Universitario de Alicante, ISABIAL, \\ Alicante, Spain
}

Belén Martínez ${ }^{1}$, Juan Martínez², Juan Antonio Casellas², José R. Aparicio²

submitted 7.12.2018

accepted after revision 22.2.2019

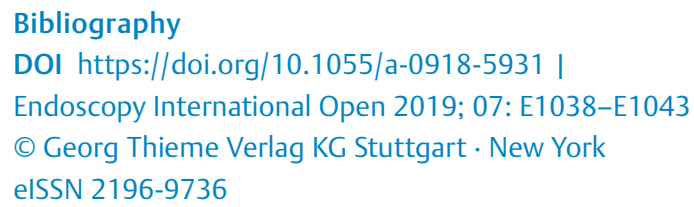

\section{ABSTRACT}

Background and study aims The aim of this study was to assess the efficacy and safety of endoscopic ultrasoundguided rendezvous (EUS-RV) for benign biliary or pancreat- ic disorders with a 22-gauge needle and a 0.018-inch guidewire.

Patients and methods Patients who underwent EUS-RV after failed biliary or pancreatic cannulation for benign disorder were candidates for this study. For EUS-RV, a 22gauge needle and a 0.018-inch guidewire were used. Inclusion criteria were unsuccessful biliary or pancreatic cannulation for therapeutic endoscopic retrograde cholangiopancreatography (ERCP) with benign biliary or pancreatic obstruction. Exclusion criteria included malignant biliary or pancreatic obstruction, inaccessible papilla due to surgically altered upper gastrointestinal anatomy or duodenal stricture, and previous sphincterotomy and/or biliary stent placement. The primary outcome was the technical success rate of biliary or pancreatic cannulation, and the secondary outcome was the rate of adverse events.

Results Thirty-one patients were evaluated. The overall technical success rate was $80.6 \%$ (81.5\% in biliary and $75 \%$ in pancreatic cases). Adverse events (AEs) were identified in $12.9 \%$ of patients, including one with biliary peritonitis, one with abdominal pain and one with severe pancreatitis plus pneumomediastinum. Only one of the AEs (3.3\%) was directly related to the rendezvous procedure.

Conclusions EUS-RV may be a safe and feasible salvage method for unsuccessful cannulation for benign disorders. Use of a 22-gauge needle with a 0.018 -inch guidewire may be the first option for benign pathology.

\section{Introduction}

Endoscopic retrograde cholangiopancreatography (ERCP) is the standard technique for endoscopic treatment of biliary and pancreatic diseases. However, selective duct cannulation is sometimes impossible, with a failed biliary cannulation rate reportedly ranging from $3 \%$ to $10 \%$. Alternative means of achieving biliary decompression include percutaneous transhepatic drainage (PTD) [1-3] and surgical intervention [4]. However, both PTD and surgical intervention are associated with significant morbidity [5-7]. EUS-RV has been increasingly reported as an alternative to the percutaneous or surgical approach for unsuccessful cannulation in ERCP [8-11]. There is no standard EUS-RV technique, and there are many guidewires that can be used; however, there are no current data on the effectiveness of one guidewire in particular over another.

Usually, a 19-gauge needle and a 0.025 - or 0.035 -inch guidewire are used. However, in cases of benign pathology, puncture of a nondilated duct with this needle can be very difficult or even impossible. In addition, in these cases, the possibility of performing transmural drainage is not an appropriate option, so if rendezvous is not successful, puncturing the duct with a large-gauge needle can result in adverse events (AEs). The most recent review revealed that the technical success 
rate of EUS-RV was $81 \%$, with an AE rate of $10 \%$, primarily in malignant indications [10]. Almost all of the reports reviewed were retrospective studies from a single high-volume center with skilled endosonographers. However, a Spanish national survey showed that the technical success rate was $68.3 \%$, with an $\mathrm{AE}$ rate of $21.6 \%$ [12].

A small guidewire of 0.018 inches could be more effective for crossing a stenosis or the papilla. This guidewire can be used with a 22-gauge needle, which facilitates puncture of the nondilated duct and could reduce AEs. Therefore, we conducted this retrospective study to evaluate the efficacy and safety of using a 0.018-inch NovaGold High Performance (Boston Scientific, Marlborough, Massachusetts, United States) guidewire for EUS-RV in patients with benign biliary or pancreatic diseases.

\section{Patients and methods}

This was a retrospective study of a prospectively updated database from two Spanish hospitals. Patients with benign biliary or pancreatic pathology who had undergone previous unsuccessful ERCP since 2016 were included. Exclusion criteria included malignant biliary or pancreatic obstruction, inaccessible papillae due to surgically altered upper gastrointestinal anatomy or duodenal stricture, and previous sphincterotomy and/or biliary stent placement. Written informed consent for ERCP and EUSguided procedures was obtained from all patients.

\section{EUS-RV}

All procedures were performed by endoscopists who were well trained and experienced with therapeutic ERCP and endoscopic ultrasound (EUS) procedures at each center. Patients received prophylactic antibiotics before the procedures, which were performed under endoscopist-directed sedation with propofol or general anesthesia. EUS-RV was performed in patients with unsuccessful biliary or pancreatic cannulation using a linear array echoendoscope (GF-UCT180; Olympus Europe, Hamburg, Germany). EUS-RV was performed during the same session after a failed ERCP or on a different day at the discretion of the endoscopist. For biliary EUS-RV, the extrahepatic bile duct (EHBD) was punctured through the first (D1) and second portions (D2) of the duodenum with long and short scope positions, respectively. Choice of puncture site was established based on endoscope stability. For pancreatic EUS-RV, the pancreatic duct was punctured through the stomach or D1. The duct was punctured using a 22-gauge needle (Expect, Boston Scientific, Marlborough, Massachusetts, United States) under Doppler imaging guidance to avoid any interposing vessels. Biliary or pancreatic access was confirmed by bile or pancreatic juice aspiration, and contrast medium was injected to evaluate the biliary system or pancreatic duct. A 0.018-inch guidewire (NovaGold; Boston Scientific, Marlborough, Massachusetts, United States) was inserted through the needle and advanced deeply into the duodenum, across the stricture and/or the papilla. After the guidewire tip was adequately placed into the duodenum, the echoendoscope was withdrawn, and the duodenoscope was positioned opposite the papilla, where the EUS-placed guidewire exits from the papilla. Subsequently, biliary or pancreatic can-

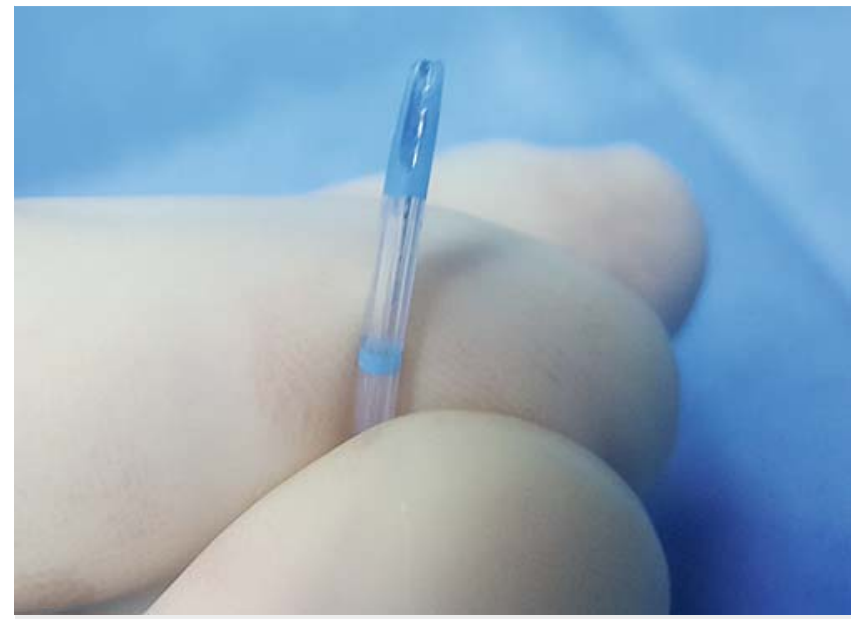

- Fig. 1 Hand-made "monorail" sphincterotome with slit at its distal end.

nulation was attempted over the EUS-placed guidewire that was grasped with a snare or biopsy forceps and pulled back into the accessory channel of the duodenoscope. Since June 2017, a manually modified "monorail" sphincterotome with a slit at its end ( $\mathbf{F i g . 1}$ ) has been used to cannulate over the guidewire. The sphincterotome used was the same as that in the index ERCP if the EUS-RV was performed in the same session. A slit was made in the convex part of the distal end of the sphincterotome with a scalpel blade. For cannulation with this monorail sphincterotome, the cannula tip is aligned to the rendezvous guidewire in the duodenum. Once the slit on the tip is snapped onto the rendezvous guidewire, the sphincterotome is advanced through the ampulla while maintaining the alignment of the rendezvous guidewire and the sphincterotome. Once the sphincterotome appears to pass the papilla, a guidewire preloaded in the sphincterotome is advanced into the bile duct, followed by sphincterotome insertion ( $\vee$ Video 1 ).

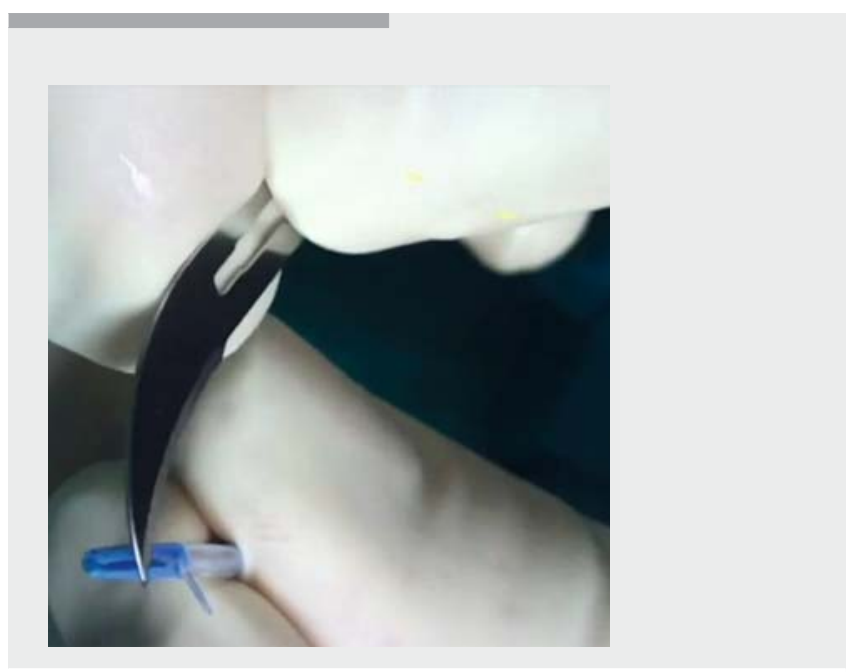

$\square$ Video 1 EUS-RV and cannulation with “monorail” sphincterotome. 


\begin{tabular}{|c|c|c|}
\hline Sex, female/male, n & $13 / 18$ & \\
\hline Age, years, mean (SD) & $71.8(13.1)$ & \\
\hline \multicolumn{3}{|l|}{ Type EUS-RV } \\
\hline - Biliary, n & 27 & \\
\hline - Pancreatic, n & 4 & \\
\hline \multicolumn{3}{|l|}{ Primary disease, $n$} \\
\hline - Bile duct stone, $\mathrm{n}$ & 20 & \\
\hline - Benign stenosis, $\mathrm{n}$ & 5 & \\
\hline - Biliary leak, n & 2 & \\
\hline - Pancreatic stenosis, $\mathrm{n}$ & 1 & \\
\hline - Pancreatic fistula, $\mathrm{n}$ & 1 & \\
\hline - Lithotripsy for pancreatic lithiasis, n & 2 & \\
\hline \multicolumn{3}{|l|}{ Approach route } \\
\hline \multicolumn{3}{|l|}{ - Pancreatic EUS-RV, n (\%) } \\
\hline - Stomach & $4(100 \%)$ & \\
\hline \multicolumn{3}{|l|}{ - Biliary EUS-RV, n (\%) } \\
\hline - Stomach & $1(3.7 \%)$ & \\
\hline - D1 & $10(37 \%)$ & \\
\hline$-\mathrm{D} 2$ & $16(59.3 \%)$ & \\
\hline Success rate (\%) & & $P=0.8$ \\
\hline - Overall & $25 / 31(80.6 \%)$ & \\
\hline - Pancreatic EUS-RV & $3 / 4(75 \%)$ & \\
\hline - Biliary EUS-RV & $22 / 27(81.5 \%)$ & \\
\hline CBD diameter, median (IQR) mm & $6(4-9)$ & \\
\hline PD diameter, median (IQR) mm & $6.5(2.5-9.75)$ & \\
\hline \multicolumn{3}{|l|}{ Adverse events } \\
\hline - Overall, n (\%) & $4(12.9 \%)$ & \\
\hline - Abdominal pain, $\mathrm{n}$ & 1 & \\
\hline - Bile leak, n & 1 & \\
\hline - Pancreatitis, n & 1 & \\
\hline - Pneumomediastinum, $\mathrm{n}$ & 1 & \\
\hline
\end{tabular}

Following successful cannulation, appropriate treatment could be performed.

The NovaGold is a 0.018 -inch guidewire with a Triton Alloy Core that provides adequate stiffness for both pushability and flexibility and a 6-cm radiopaque atraumatic tip and 30-cm hydrophilic coating.

\section{Outcomes}

The primary outcome was the technical success rate of selective biliary or pancreatic cannulation, and the secondary outcome was the rate of AEs. AEs were defined according to the severity grading system of the American Society for Gastrointestinal Endoscopy lexicon.

\section{Statistical analysis}

Continuous variables are presented as median or mean values. The Fisher exact test and Mann-Whitney $U$ test were used for comparison of categorical and continuous variables, respectively. Differences with a $P<0.05$ were considered statistically significant. All data were analyzed with IBM SPSS statistics software version 20.0 for Macintosh.

\section{Results}

From March 2016 to July 2018, 460 ERCPs were performed for benign biliary or pancreatic pathology. Of these, 31 EUS-RV procedures were performed after unsuccessful cannulation (6.7\%) on 18 men and 13 women with an average age (standard deviation) of 71.8 years (13.1). In 27 cases, the EUS-RV was biliary, and in four cases, it was pancreatic. - Table 1 shows characteristics of the procedures performed. Initial ERCP was performed for bile duct stones $(n=20)$, benign stenosis $(n=5)$, biliary leak $(n=2)$, pancreatic stenosis $(n=1)$, pancreatic fistula $(n=1)$, and treatment of intraductal lithiasis in chronic pancreatitis $(n=2)$. Twenty-five patients $(80.6 \%)$ underwent EUS$R V$ in the same session after failed ERCP. Reasons for applying EUS-RV were undetectable papilla $(n=3)$, intradiverticular papilla $(n=9)$, and failed cannulation $(n=19)$ that could not be achieved even with use of advanced techniques such as the double guidewire method, transpancreatic sphincterotomy or needle-knife precut papillotomy. In three of these cases, EUS$R V$ was performed as a rescue method after an AE during the ERCP: two retroperitoneal perforations after transpancreatic sphincterotomy and one free perforation after a resection of high-grade dysplasia adenoma in the papillary area. In the three cases, EUS-RV was successfully performed, the programmed therapy was completed, and the patients were discharged the next day.

For pancreatic EUS-RV, access to the pancreatic duct was through the stomach in all cases. For biliary EUS-RV, access to the common bile duct was from the gastric antrum in one case, D1 in 10 cases and D2 in 16 cases, so the position of the endoscope for biliary EUS-RV was in the long position in 11 cases $(40.7 \%)$ and in the short position in 16 cases (59.3\%).

The median (interquartile range [IQR]) of the common bile duct diameter was $6 \mathrm{~mm}$ (range $4-9 \mathrm{~mm}$ ) and for the pancreatic duct, it was $6.5 \mathrm{~mm}$ (range $2.5-9.75 \mathrm{~mm}$ ). Median (IQR) of the common bile duct diameter tended to be lower in patients in whom the rendezvous was successful: $5.5 \mathrm{~mm}(4-7.5)$ vs. $7 \mathrm{~mm}(4.5-14.5)$, although the differences were not significant, $P=0.3$.

Duct puncture and contrast injection were achieved with the 22-gauge needle in all cases. However, the guidewire could be 
placed only in the duodenum in 25 of 31 patients (80.6\%), completing the procedure in all cases in which the guide was passed, which represents a technical and clinical success rate of $80.6 \%$. There were no cases of shearing the guidewire during its handling. However, when the guidewire is manipulated for a long time, the tip tends to bend, which makes it difficult to handle and could have been the cause of the six failures.

In pancreatic EUS-RV, the technical success rate was $75 \%$ (3/4), and in biliary EUS-RV, it was $81.5 \%$ (22/27). Among the six cases of unsuccessful EUS-RV, one patient required surgery; in two cases, no other procedure was performed, and in three cases, a new EUS-RV trial was performed with a 19-gauge needle and a 0.025-inch VisiGlide (Olympus, Tokyo, Japan) guidewire. In all three cases, the new EUS-RV was completed, so the overall success rate of the EUS-RV for these benign cases was 28 of $31(90.3 \%)$.

The overall $\mathrm{AE}$ rate was $12.9 \%$ (4 AE in 3 patients), all of which were in biliary EUS-RV. AEs included self-limiting abdominal pain in one case, biliary peritonitis that required surgery in one patient who failed to perform the EUS-RV, and severe pancreatitis in one patient who underwent transpancreatic sphincterotomy during initial ERCP. This patient presented with severe desaturation and subcutaneous emphysema after the procedure and was diagnosed tracheal laceration and pneumomediastinum in relation to orotracheal intubation.

AEs were mild in $3.2 \%$ of cases and severe in $9.8 \%$ : One case required surgery, and the remaining three cases were managed conservatively. In the patient who presented with bile leak and biliary peritonitis, we tried to introduce an ERCP catheter into the common bile duct to manipulate the guidewire. However, that was not achieved due to absence of dilatation of the common bile duct. After this $A E$, no further manipulation of the guidewire with a catheter was attempted in any case.

In 19 cases (61.3\%), a monorail sphincterotome was used, which was useful in 15 cases (78.9\%). In the cases in which the monorail sphincterotome was effective, total time of the exploration was considerably reduced: 29.5 minutes (20.3 - 35.5) vs. 89 minutes $(41-120), P=0.008$.

There were no significant differences in terms of the influence of access route on the technical success rate or on the rate of AEs between D1 and D2: $81.8 \%$ vs. $81.2 \%(P=1)$ and $9.1 \%$ vs. $12.5 \%(P=0.8)$, respectively.

\section{Discussion}

EUS-RV was first reported by Mallery et al. [13] and is now an effective salvage technique for accessible papilla and unsuccessful cannulation [8-11,14-16]. Based on their review of 382 cases of EUS-RV from 15 published reports, Tsuchiya et al. [10] reported an overall success rate of $82 \%$, with a $13 \%$ incidence of AEs, mainly bleeding, bile leakage, peritonitis, pneumoperitoneum, and pancreatitis. Some of these AEs clearly may be related to the caliber of the needle used. However, the studies included in this review described use of EUS-RV mostly for unresectable malignant biliary disorders. - Table 2 shows the studies with cases of rendezvous in benign pathology.
- Table 2 Previous studies with cases of rendezvous in benign pathology.

\begin{tabular}{|l|r|l|l|}
\hline Study & $\mathbf{n}$ & Technical success & Adverse events \\
\hline Kahaleh [22] & 6 & $5 / 6(83 \%)$ & $1 / 6(16 \%)$ \\
\hline Brauer [24] & 4 & $3 / 4(75 \%)$ & $1 / 4(25 \%)$ \\
\hline Kim [21] & 5 & $3 / 5(60 \%)$ & $1 / 5(20 \%)$ \\
\hline Dhir [16] & 15 & $15 / 15(100 \%)$ & $0 / 15(0 \%)$ \\
\hline Kawakubo [20] & 9 & $9 / 9(100 \%)$ & $1 / 9(11 \%)$ \\
\hline Iwashita [8] & 8 & $7 / 8(87 \%)$ & $1 / 8(12 \%)$ \\
\hline Shiomi [18] & 12 & $10 / 12(83 \%)$ & $2 / 12(16 \%)$ \\
\hline Current study & 31 & $28 / 31(90.3 \%)$ & $4 / 31(12.9 \%)$ \\
\hline
\end{tabular}

In most studies, EUS-RV was performed with a 19-gauge needle and a 0.035 - or 0.025 -inch guidewire. However, in benign disorders, it may be difficult even for skilled endosonographers to perform a 19-gauge needle puncture of a nondilated duct. Therefore, smaller needles and guidewires can be used, with greater ease for puncture of the nondilated duct and theoretically with fewer AEs, since in case of failure, transmural drainage would not be a rescue option. In our study, only one $\mathrm{AE}$ (bile leak) was clearly related to the procedure and was most likely due to the attempt to manipulate the guidewire with a catheter.

Although EUS-RV is now performed relatively routinely in a few high-volume centers, standardization of procedures and development of exclusive devices for EUS-RV remain underway; therefore, the procedure is not yet widely used.

For this reason, we retrospectively evaluated efficacy and safety of EUS-RV for benign disorders with use of a 22-gauge needle and a 0.018 -inch guidewire. For such cases, we report an overall technical success rate of $80.6 \%$, with an $A E$ rate of $12.9 \%$; these rates are comparable to those of previously published studies in malignant biliary disorders [10]. In comparison with EUS-transluminal biliary drainage, the clinical advantages of EUS-RV include avoidance of creation of a permanent bilioenteric fistula and a fistulous tract dilation, which leads to less bile leakage. Therefore, EUS-RV should be the procedure of choice for benign disorders.

Guidewire manipulation is an important step for EUS-RV success. In our study, EUS-RV was unsuccessful in 6 patients. Failure to advance the guidewire into the duodenum was the cause of unsuccessful EUS-RV in all 6 cases. Shah et al. [17] reported a $75 \%$ EUS-RV success rate, with difficult guidewire manipulation identified as the primary reason for unsuccessful EUS-RV.

Shiomi et al. [18] reported an $85 \%$ EUS-RV success rate for benign and resectable malignant biliary disorders, with a $15 \%$ incidence of AEs, including two cases of biliary peritonitis. In this study, all cases were performed with a 19-gauge needle and a 0.025 -inch guidewire. Although the results are similar to ours, there was a greater number of AEs directly related to the procedure: $10 \%$ biliary leaks vs. $3.2 \%$. This small study included 
only 20 patients with benign (12 patients) and resectable malignant biliary disorders (8 patients) from 12 hospitals, so very few cases occur in each hospital. In contrast, our study focuses exclusively on the utility of EUS-RV in benign pathology. The results with pancreatic EUS-RV are worse, with a $64.7 \%$ technical success rate in one study [19].

The major AEs associated with EUS-RV are bleeding, biliary peritonitis, pancreatitis, and perforation $[10,11,14,16,20-$ 23]. In our study, the overall adverse AE was $12.9 \%$, including only one case of biliary peritonitis. This severe $A E$ is due to bile leakage through the puncture site, and it has been associated with unsuccessful EUS-RV and attempts to manipulate the guide with a catheter. After this case, we did not use the catheter again, and there were no new cases of biliary peritonitis. The other AEs may be related to the previous ERCP attempt.

Although Iwashita et al. $[8,14]$ show that the D2 route is the most appropriate route for EUS-RV because of the short distance to the papilla from the puncture site and ease of guidewire manipulation, our results show no difference. This finding is probably related to ease of use of the 22-gauge needle, both in D1 and in D2, and although the handling of the guidewire from D1 is more complex, the smaller caliber of the 0.018-inch guidewire, together with its rigidity, allows easy passage through the papilla in most situations.

As mentioned above, although some studies have shown that the efficacy of EUS-RV in failed ERCP, no study has directly compared the outcome of benign and malignant disorders. Shiomi et al [18] reviewed previous studies consisting of more than 10 cases that described the success rates and AEs resulting from EUS-RV for benign and malignant biliary disorders. A success rate of $88 \%$ was achieved in the benign group compared to that of $92 \%$ in the malignant group. The $A E$ rate was $11 \%$ in both groups. As a result, EUS-RV appears to be a suitable intervention after failed biliary cannulation for benign biliary disorders. However, there is still insufficient evidence to determine which approach route is best suited for benign conditions or malignancy. Based on the results of our study, in patients with benign pathology and unsuccessful cannulation, use of a 22gauge needle and a 0.018-inch NovaGold guidewire may be the appropriate choice, with a high success rate and minimal AEs if only the duct is punctured with the needle. In the case of failure, there is always the opportunity to attempt the puncture with a 19-gauge needle and a 0.025 -inch guidewire; this approach becomes easier once the duct has been dilated with a previous contrast injection.

Recently, Nakai et al. [24] showed the benefit of a novel "hitch-and-ride catheter" for biliary cannulation after EUS-RV. They used this catheter successfully after EUS-RV in 11 cases of biliary cannulation, thus saving a significant amount of time. We used a manually modified sphincterotome with a scalpel blade, making a small slit in the convex part of the sphincterotome end ( $\nabla$ Fig.1) ( $\downarrow$ Video 1 ). All cases used the same sphincterotome as was used in ERCP. We used this device in 19 patients; we found it to be useful in 15 patients (78.9\%), and it significantly reduced total procedure time.

The limitations of our study should be acknowledged. The study was a single-arm retrospective study. A randomized con- trolled trial comparing other needles and guidewires is needed to overcome these limitations.

\section{Conclusion}

In conclusion, we recommended EUS-RV with a 22-gauge needle and a 0.018-inch NovaGold guidewire for unsuccessful biliary cannulation for benign biliary and pancreatic disorders. In the future, standardization of the EUS-RV technique should be extensively evaluated in clinical trials.

\section{Competing interests}

None

References

[1] Ferrucci JT Jr., Mueller PR, Harbin WP. Percutaneous transhepatic biliary drainage: technique, results, and applications. Radiology 1980; 135: $1-13$

[2] Harbin WP, Mueller PR, Ferrucci JrJT. Transhepatic cholangiography: complications and use patterns of the fine-needle technique: a multiinstitutional survey. Radiology 1980; 135: 15-22

[3] Calvo MM, Bujanda L, Heras I et al. The rendezvous technique for the treatment of choledocholithiasis. Gastrointest Endosc 2001; 54: $511-513$

[4] Smith AC, Dowsett JF, Russell RC et al. Randomised trial of endoscopic stenting versus surgical bypass in malignant low bile duct obstruction. Lancet 1994; 344: 1655-1660

[5] Laméris JS, Stoker J, Nijs HG et al. Malignant biliary obstruction: percutaneous use of self-expandable stents. Radiology 1991; 179: $703-707$

[6] Beissert M, Wittenberg G, Sandstede J et al. Metallic stents and plastic endoprostheses in percutaneous treatment of biliary obstruction. Z Gastroenterol 2002; 40: $503-510$

[7] Kama NA, Coskun T, Yuksek YN et al. Factors affecting post-operative mortality in malignant biliary tract obstruction. Hepatogastroenterology 1999; 46: $103-107$

[8] Iwasitha T, Yasuda I, Mukai T et al. EUS-guided rendezvous for difficult biliary cannulation using a standardized algorithm: a multicentre prospective pilot study (with videos). Gastrointest Endosc 2016; 83: $394-400$

[9] Barkay O, Sherman S, McHenry L et al. Therapeutic EUS-assisted endoscopic retrograde pancreatography after failed pancreatic duct cannulation at ERCP. Gastrointest Endosc 2010; 71: 1166 - 1173

[10] Tsuchiya T, Itoi T, Sofuni A et al. Endoscopic ultrasonography-guided rendezvous technique. Dig Endosc 2016; 28: 96 - 101

[11] Dhir V, Bhandari S, Bapat M et al. Comparison of EUS-guided rendezvous and precut papillotomy techniques for biliary access. Gastrointes Endosc 2012; 75: 354 - 359

[12] Vila JJ, Pérez-Miranda M, Vazquez-Sequeiros E et al. Initial experience with EUS-guided cholangiopancreatography for biliary and pancreatic duct drainage: a Spanish national survey. Gastrointest Endosc 2012; 76: $1133-1141$

[13] Mallery S, Matlock J, Freeman ML. EUS-guided rendezvous drainage of obstructed biliary and pancreatic ducts: report of 6 cases. Gastrointest Endosc 2004; 59: 100-107 
[14] Iwashita T, Lee JG, Shinoura S et al. Endoscopic ultrasound-guided rendezvous for biliary access after failed cannulation. Endoscopy 2012; 44: $60-65$

[15] Isayama H, Nakai Y, Kawakubo K et al. The endoscopic ultrasonography-guided rendezvous technique for biliary cannulation: a technical review. J Hepatobiliary Pancreat Sci 2013; 20: 413-420

[16] Dhir V, Artifon EL, Gupta K etal. Multicenter study on endoscopic ultrasound-guided expandable biliary metal stent placement: choice of access route, direction of stent insertion, and drainage route. Dig Endosc 2014; 26: 430-435

[17] Shah JN, Marson F, Weilert F et al. Single-operator, single-session EUSguided anterograde cholangiopancreatography in failed ERCP or inaccessible papilla. Gastrointest Endosc 2012; 75: 56-64

[18] Shiomi H, Yamao K, Hoki N et al. Endoscopic ulstrasound-guided rendezvous technique for failed biliary cannulation in benign and resectable malignant biliary disorders. Dig Dis Sci 2018; 63: 787-796

[19] Kurihara T, Itoi T, Sofuni A et al. Endoscopic ultrasonography-guided pancreatic duct drainage after failed endoscopic retrograde cholangiopancreatography in patients with malignant and benign pancreatic duct obstructions. Dig Endosc 2013; 25: 109-116
[20] Kawakubo K, Isayama H, Sasahira N et al. Clinical utility of an endoscopic ultrasound-guided rendezvous technique via various approach routes. Surg Endosc 2013; 27: 3437-3443

[21] Kim YS, Gupta K, Mallery S et al. Endoscopic ultrasound rendezvous for bile duct access using a transduodenal approach: cumulative experience at a single center. A case series. Endoscopy 2010; 42: 496 502

[22] Kahaleh M, Hernandez A], Tokar J et al. Interventional EUS-guided cholangiography: evaluation of a technique in evolution. Gastrointest Endosc 2006; 64: 52 - 59

[23] Khashab MA, Valeshabad AK, Modayil R et al. EUS-guided biliary drainage by using a standardized approach for malignant biliary obstruction: rendezvous versus direct transluminal techniques (with videos). Gastrointest Endosc 2013; 78: 734 - 741

[24] Nakai Y, Isayama H, Matsubara S et al. A novel "hitch-and-ride" deep biliary cannulation method during rendezvous endoscopic ultrasound-guided ERCP technique. Endoscopy 2017; 49: 983 - 988

[25] Brauer BC, Chen YK, Fukami N et al. Single-operator EUS-guided cholangiopancreatography for difficult pancreatobiliary access. Gastrointest Endosc 2009; 70: 471-479 